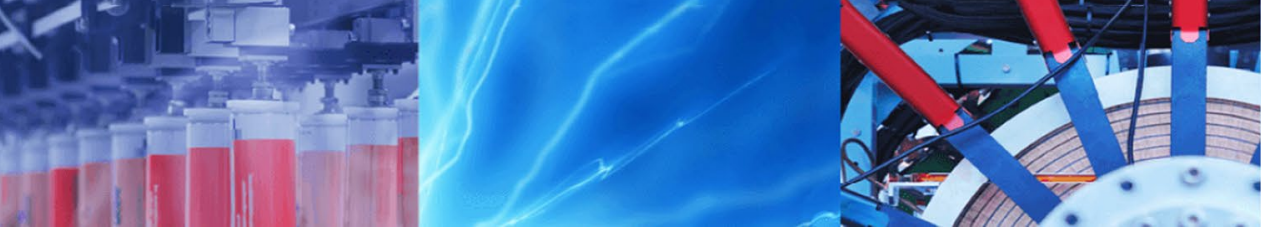

Research Article

\title{
Step-graded InAsP buffer layers with gradient interface grown via metal organic chemical vapor deposition
}

\author{
Jian $\mathrm{Li}^{1,2} \cdot \mathrm{Xin} \mathrm{Wei}^{1,2}$ (D) \\ (c) Springer Nature Switzerland AG 2019
}

\begin{abstract}
A highly mismatched InGaAs metamorphic layer is grown by introducing a gradient interface between step-graded InAsP buffer layers via metalorganic chemical vapor deposition. The crystal growth quality of the gradient interface metamorphic layers and the one without gradient interface are compared. According to the tested results, applying a gradient interface between the step-graded InAsP buffer layers halves the surface roughness and doubles the photoluminescence intensity of the epilayers. This proves that the introduction of the gradient interface can improve the crystal quality and enhance the dislocation fusion during the growth of the large-lattice-mismatch materials.
\end{abstract}

Keywords InGaAs metamorphic growth · Step-graded buffers · Gradient interface (GI)

\section{Introduction}

The short-wavelength infrared (SWIR 1-3 $\mu \mathrm{m}$ ) is a very important spectrum range for imaging and sensing. For wavelength less than $1.7 \mu \mathrm{m}$, lattice-matched InGaAs(P)/ InP materials have been fully developed and prove to be the best candidate for both photodetectors and lasers. To extend the wavelength of InGaAs beyond $2 \mu \mathrm{m}$, the In content of the InGaAs material must be increased. As a result, the lattice constant of the InGaAs will significantly increase, which in turn increases the lattice mismatch between $\ln \mathrm{GaAs}$ and the $\mathrm{InP}$ substrate. As the lattice mismatch increases, the dislocations are also increased and the material quality of the InGaAs decreases rapidly.

The introduction of a buffer layer has been proved to be an efficient method to filter the dislocations and increase the crystal growth quality. The buffer layers are classified according to their material systems, such as ternary materials, e.g., InGaAs [1, 2], InAlAs [3, 4], and InAsP [5, 6], and quaternary materials, e.g., InAIGaAs [7, 8], InGaAsP [9], and AIGaAsSb [10]. The ternary InAIAs and InAsP are the commonly used InP-based buffer layer materials. The presence of $\mathrm{Al}$ in the buffer layer constrains the improvement in crystal quality in the InAIAs buffer layers. On the contrary, the double-anion (group $V$ atoms) characteristic of InAsP offers the potential advantages of easy growth rate and alloy composition control compared with the double-cation (group III atoms)-based materials. In addition, the lattice constant and energy bandgap of InAsP span between InP and InAs, which makes it an ideal buffer material for the growth of InAs quantum well/dot lasers and expanded-wavelength InGaAs photodetectors.

There are several growth options for the buffer layers, such as the step-graded buffer layers [11-13], lineargraded buffer layers [14], two-/multi-step low-temperature buffer layers [15, 16], and superlattice buffer layers [17]. Linear-graded buffer layers are commonly used in metalorganic chemical vapor deposition (MOCVD) growth of the buffer layers: By linearly adjusting the source flow rate with the mass flow controller (MFC), the material composition of the buffer layer can be easily changed from the substrate to the desired composition of the epitaxial layer. Since the dislocations are merged through the extension of the linear-graded buffer, it is necessary to find a suitable

$\triangle$ Xin Wei, weix@semi.ac.cn | ${ }^{1}$ Institute of Semiconductors, Chinese Academy of Sciences, Beijing 100083, China. ${ }^{2}$ College of Materials Science and Opto-Electronic Technology, University of Chinese Academy of Sciences, Beijing 100049, China.

SN Applied Sciences (2019) 1:585 | https://doi.org/10.1007/s42452-019-0606-1

Received: 5 January 2019 / Accepted: 13 May 2019 / Published online: 17 May 2019 
gradation speed for a better control of the dislocation density in epitaxial layers. Unlike the linear-graded method, the dislocations in the step-graded buffer are merged around the interface between two neighboring buffer layer steps. Hence, the control of the buffer layer interface is essential to reduce the dislocations in the step-graded buffer layers. The previous work has demonstrated that to grow a step buffer before the linear grading buffer is benefit to the crystal quality [18]. In our experiment, we have combined the step-graded buffer layer with the linear-graded buffer layer. By introducing a linearly composition-graded gradient interface (GI) between the two neighboring step buffer layers, the step-graded buffer layer is linearly graded from one composition to the next composition. We find that the introduction of the $\mathrm{Gl}$ can change the dislocation slip mode at the step-graded buffer layer interface and can be used to suppress the extension of the dislocations in the buffer layer.

A preliminary theoretical discussion of the factors that influence the dislocation formation during the buffer layer growth will be followed by the experimental details in the subsequent sections. In the Results and discussion section, we will prove the benefit of introducing a Gl between the neighboring steps of the graded buffers using various test methods.

\section{Theory}

During epitaxy growth, if the thickness of the epitaxial layer exceeds the critical thickness of the current strain situation, the stress generated from the lattice mismatch is released in the form of dislocations. These dislocations subsequently spread, slip, and merge with the continual growth of the epitaxial layers to achieve a stable state [19]. The dislocation density in the step-graded buffer layers can be expressed by the following empirical formula proposed by Fitzgerald et al. [20]:

$\rho_{\mathrm{t}}=\frac{2 R_{\mathrm{g}} R_{\mathrm{gr}} e^{\frac{u}{k T}}}{|b| B Y^{m} \varepsilon_{\mathrm{eff}}^{m}} \propto \frac{R_{\mathrm{g}} R_{\mathrm{gr}}}{V}$

where $\rho_{\mathrm{t}}$ is the threading density that influences the photoluminescence $(\mathrm{PL})$ intensity and $\mathrm{X}$-ray diffraction (XRD) full width half maximum (FWHM); $B$ is a constant with a unit of velocity, $Y$ is the Young's modulus, $\varepsilon_{\text {eff }}$ is the strain reduced by the dislocation flow, $\mathrm{m}$ is an exponent between 1 and $2, R_{\mathrm{g}}$ is the growth rate, $R_{\mathrm{gr}}$ is the variation rate (lattice mismatch per unit thickness), $U$ is the dislocation glide activation energy, $k$ is the Boltzmann constant, $T$ is the temperature, and $V$ is the dislocation slip velocity, which can be expressed as follows:
$V=B Y^{m} \varepsilon_{\mathrm{eff}}^{m} e^{-\frac{u}{k T}}$

From the above equation, we see that during the growth of the step-graded buffer layers, the threading dislocation (TD) density is proportional to the growth rate and the variation rate and inversely proportional to the material-determined dislocation slip velocity expressed as $V$. Therefore, by changing the variation rate $\left(R_{\mathrm{g}}\right)$, growth rate $\left(R_{\mathrm{gr}}\right)$, and the dislocation slip velocity $(V)$, the dislocation density in the buffer layer can be effectively controlled. Since the dislocation slip velocity is determined by the material properties and strains, in order to optimize the growth quality of the buffer layer, we need to calculate the interlayer mismatch between the steps of the graded buffer layer. Romanov and Speck et al. used a series of firstorder nonlinear partial differential equations to describe the process of annihilation through which the dislocations merge and evolve [21]. According to their research, sufficient annihilation of the dislocations, without generating new dislocations, can be ensured, if the initial mismatch between the epitaxial layers does not exceed 0.003 .

In the step-graded buffer layer, the composition of the material changes abruptly from one graded layer to another. Generally, it is believed that the mismatch stress is released at the interface between the buffer layers and new dislocations are formed if the growth condition is not well controlled. Moreover, while the TD is expected to decrease, it actually increases when the newly generated dislocations are more than expected. As a result, the material quality significantly decreases although the grown buffer layer is sufficiently thick. Therefore, it is essential to control the generation and fusion of dislocations at the interface of the step-graded buffer layer to reduce the overall dislocation level of the buffer layer. Fortunately, during the growth of the linear-graded buffer, a dislocation slip can be achieved by changing the graded gradient of the linear-graded buffer. We introduced the lineargraded buffer layer at the interface of the step-graded buffer as a Gl to control the extent of dislocations formed at the interface of the step-graded buffer and proved its effectiveness using a series of experiments.

\section{Experimental details}

All samples in this paper were grown on 2-inch (001)-oriented InP epi-ready substrates using the Aixtron AIX-200 MOCVD system. The pressure in the reactor chamber was maintained at 20 mbar. Using purified $\mathrm{H}_{2}$ as carrier gas, the group III sources trimethylindium (TMIn), trimethylgallium (TMGa) and group $\mathrm{V}$ sources pure arsine $\left(\mathrm{AsH}_{3}\right)$ and pure phosphine $\left(\mathrm{PH}_{3}\right)$ were injected into the reactor 
chamber. Before the epilayer growth, the substrate was heated to $655^{\circ} \mathrm{C}$ and maintained at this temperature for 10 min in $\mathrm{PH}_{3}$ atmosphere as a pretreatment to remove the surface oxide on the epi-ready substrate. An InP buffer layer was also grown under this temperature before reducing the temperature to $625^{\circ} \mathrm{C}$ to grow the InAsP buffers. The growth rate of the InAsP buffer was maintained at about $1.35 \mu \mathrm{m} / \mathrm{h}$. During the growth, the ratio between the group $V$ and group III sources (V/III) was maintained at $70 \pm 2$. The step-graded ratio was about $0.01 / \mu \mathrm{m}$, and the gradient ratio of the 12-nm-thick gradient interface was set to $0.25 / \mu \mathrm{m}$.

Since InAsP is a double-anion-based material, its growth rate can be easily controlled by directly controlling the flow rate of the In source. At the same time, the composition of InAsP can be easily controlled by adjusting the flow rate of the group $V$ sources to adjust the lattice constant of the epitaxy layer. In order to determine the growth thickness of the buffer layer step, the critical thickness between the two adjacent epitaxy InAsP buffer layers was calculated using J. Zou's method [22] with a strain step of 0.003 . The results are listed in Table 1.

Previous experimental and theoretical calculations showed that for III-V-V-type ternary materials, the growth rate is mainly determined by the group III atoms and the group $V$ sources are excessive in the reaction chamber. Moreover, the gas-phase composition and solid-phase composition of the group $V$ atoms in the reaction chamber are different [23]. We designed a series of composition calibration growth experiments to determine the flow rate of $\mathrm{AsH}_{3}$ and $\mathrm{PH}_{3}$ in the growth process of different InAs $1-y$ P $\mathrm{P}_{\mathrm{y}}$ compositions. The experiments were divided into two categories. The first is the epitaxy growth under smallstrain conditions: Seven samples of five-period InP/InAsP superlattice with different compositions were grown, and the strained compositions of these materials were calculated by analyzing the XRD fringe peaks of the superlattice. The second category was the epitaxy growth under large-strain conditions. Five samples of 1 - $\mu \mathrm{m}$-thick InAsP monolayers were grown, and the relaxed compositions of these materials were also calculated by XRD peak position analysis. All calculated data points are plotted in Fig. 1. The relationship between the solid and gas components can be obtained by a polynomial fitting of these points. The data points in the green circle in Fig. 1 are observed to

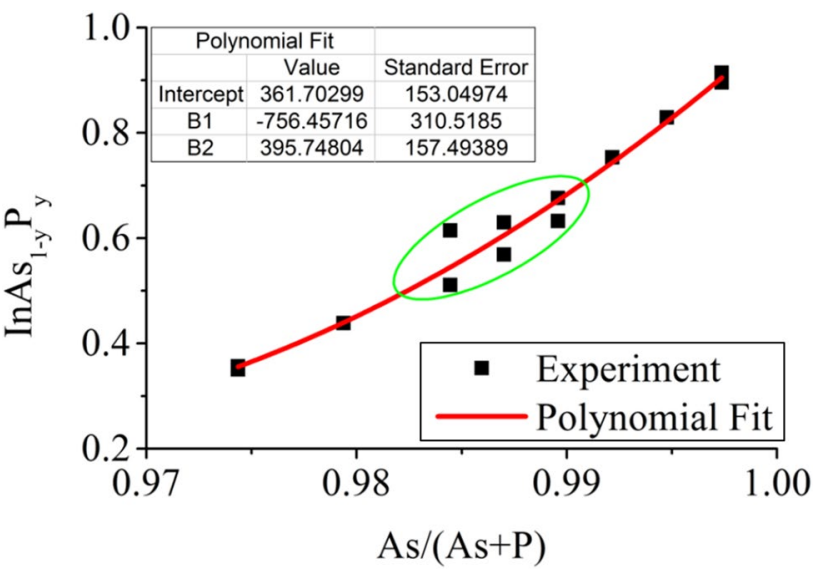

Fig. 1 Pre-experiment results and polynomial fit of the result data

deviate from the fitting curve. This is because during the growth process, when the single-layer strain is too high, the critical thickness becomes too small to perform an XRD analysis of the epitaxy layer in the superlattice structure. On the other hand, when the single-layer strain is too small, the critical thickness increases to the extent that a $1-\mu \mathrm{m}$ layer cannot sufficiently relax the epitaxy layer for XRD analysis.

After determining the relationship between the flow rate and composition, we performed the buffer growth experiment: Three different groups of buffer layer structures named S11, S12, and S13 were grown. Sample S11 contains eight buffer layers, each with a thickness of $300 \mathrm{~nm}$, which is represented using solid lines in Fig. 2a. There are 12 layers in S12 and S13, and they are represented using both the solid and dotted lines in Fig. 2a. The first four layers are divided by the dotted lines to form eight layers, and the next four layers are the same as S11. The As contents of the first seven layers of S11 were carefully adjusted to approximately $0.09,0.18,0.27,0.36$, $0.45,0.54,0.63$. A small amount of overshoot [24] was introduced in the last layer such that the As content of the InAsP was 0.65. The last four layers of $\mathrm{S} 12$ and $\mathrm{S} 13$ have the same thickness $(300 \mathrm{~nm})$ and As composition as S11. However, the thickness of their first eight layers is half that of S11 (i.e., $150 \mathrm{~nm}$ ), while the As content of each layer in $\mathrm{S} 12$ and $\mathrm{S} 13$ is half that of the corresponding layer in S11, as shown in Table 2. Meanwhile, Gls were introduced in
Table 1 Critical thickness of InAsP

\begin{tabular}{lccccccl}
\hline As mole fraction & 0.0926 & 0.1851 & 0.2777 & 0.3702 & 0.4627 & 0.5553 & 0.6478 \\
Critical thickness $(\mathrm{nm})$ & 263.8 & 264.7 & 265.7 & 266.6 & 267.5 & 268.5 & 269.4 \\
\hline
\end{tabular}

The calculation of the critical thickness is based on the neighboring content of As. For example, the critical thickness result for Column 2 (0.1851) is calculated using the mismatch between 0.1851 and 0.0926 , and the result for Column $3(0.2777)$ is calculated using the mismatch between 0.2777 and 0.1851 , and so on 


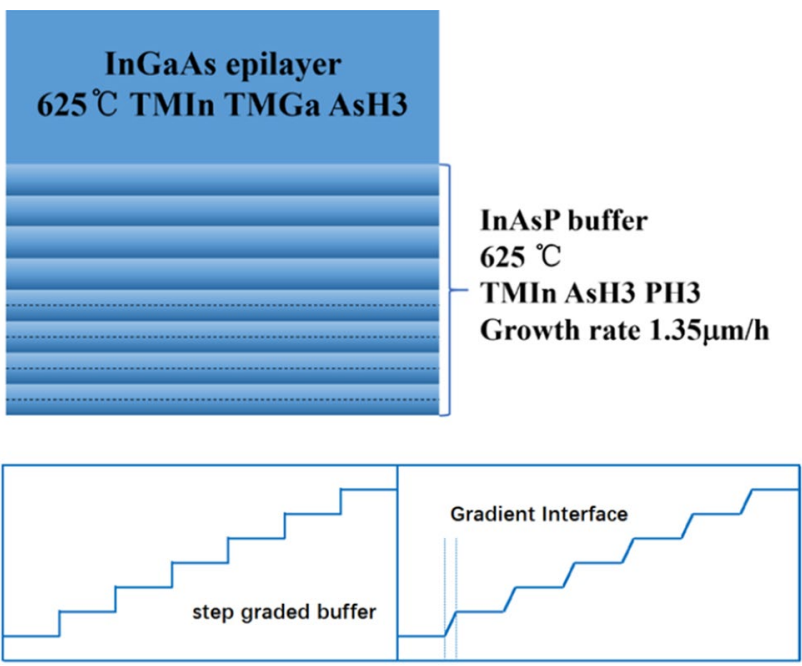

Fig. 2 a Schematic of the experiment samples. b Buffer profile of the step-graded sample (left) and the Gl sample (right)

Table 2 As content of different layers of S11, S12, and S13

\begin{tabular}{|c|c|c|c|c|}
\hline \multirow[t]{2}{*}{ Layer } & \multicolumn{2}{|l|}{ Thickness } & \multicolumn{2}{|c|}{ As content } \\
\hline & $\mathrm{S} 11(\mathrm{~nm})$ & $\begin{array}{l}\text { S12 and S13 } \\
(\mathrm{nm})\end{array}$ & $\mathrm{S} 11$ & $\mathrm{~S} 12$ and $\mathrm{S} 13$ \\
\hline \multirow[t]{2}{*}{1} & 300 & 150 & 0.09 & 0.045 \\
\hline & & 150 & & 0.09 \\
\hline \multirow[t]{2}{*}{2} & 300 & 150 & 0.18 & 0.135 \\
\hline & & 150 & & 0.18 \\
\hline \multirow[t]{2}{*}{3} & 300 & 150 & 0.27 & 0.225 \\
\hline & & 150 & & 0.27 \\
\hline \multirow[t]{2}{*}{4} & 300 & 150 & 0.36 & 0.315 \\
\hline & & 150 & & 0.36 \\
\hline 5 & 300 & 300 & 0.45 & 0.45 \\
\hline 6 & 300 & 300 & 0.54 & 0.54 \\
\hline 7 & 300 & 300 & 0.63 & 0.63 \\
\hline 8 & 300 & 300 & 0.65 & 0.65 \\
\hline
\end{tabular}

sample S13, which means that in the growth process, the interface region of the step buffer layers of S13 is grown without interruption by the linear-graded method. The gradient of the $\mathrm{Gl}$ is about $0.25 / \mu \mathrm{m}$. After the growth of the buffer layers was complete, an InGaAs epitaxy layer of approximately $1.5-\mu \mathrm{m}$ thickness was grown, in order to compare the growth quality of the buffer layers.

\section{Results and discussion}

The XRD intensity and FWHM of the grown samples are listed in Table 3. The TD density can be approximately calculated by the following equation [25]:
Table 3 Growth parameters of the three samples

\begin{tabular}{llll}
\hline ID & S11 & S12 & S13 \\
\hline Steps (thickness/nm) & $8(300 \mathrm{~nm})$ & $\begin{array}{c}8(150 \mathrm{~nm})+4 \\
(300 \mathrm{~nm})\end{array}$ & $\begin{array}{l}8(150 \mathrm{~nm})+4 \\
(300 \mathrm{~nm})\end{array}$ \\
Gradient interface & No & No & Yes \\
XRD intensity & 1306 & 1800 & 2558 \\
Substrate FWHM/arcs & 55.7 & 74.2 & 68.8 \\
Layer FWHM/arcs & 995.7 & 905.7 & 745.7 \\
\hline
\end{tabular}

$D=\frac{\mathrm{FWHM}^{2}}{2 \pi b^{2} \ln 2} \approx \frac{\mathrm{FWHM}^{2}}{4.35 b^{2}}$

where $D$ is the TD density, $b$ is the Burgers vector, and FWHM is the full width half maximum of the epilayer.

Table 3 shows that peak intensity and the FWHM of S11 are different from those of S12. This is in contradiction with the theoretical analysis that the TD density should be constant when the growth rate and the variation rate are not changed. We believe that this difference is mainly caused by the difference in the dislocation slip velocity between the two samples. The dislocation activation energy of InAs (1.4 eV) [26] is slightly less than that of $\ln P(1.6 \mathrm{eV})$ [27], and the total activation energy in the epitaxial layer decreases with As content, resulting in an increase in the slip velocity, which reduces the dislocation density and narrows the XRD peak width. After applying the $\mathrm{Gl}$, both the intensity and FWHM of S13 are observed to improve, which demonstrates the improvement in the crystal quality of the epitaxial layer.

Scanning electron microscope (SEM) images of the three samples are shown in Fig. 3. Under the same magnification, $\mathrm{S} 11$ contains obvious TD points while the other two do not. Besides, S11 and S12 contain deeper stress lines than $\mathrm{S13}$, which is the benefit of applying $\mathrm{Gl}$.

To further compare the difference between the materials with and without $\mathrm{Gl}$, we prepared two more samples named S21 and S22. The buffer structure of these two samples was identical except that GI was applied to the interface between the different steps of S22. Figure 4 shows the AFM images of S21 and S22. The calculated surface roughness of S22 is $2.16 \mathrm{~nm}$ which is almost half that of S22 (4.1 nm). By comparing the AFM image of the crosshatch channel regions, which is illustrated in the left part of Fig. 4, we observe that the trend of dislocation fusion in S22 is more obvious than in S21, which does not have $\mathrm{Gl}$. This shows that the introduction of the $\mathrm{Gl}$ improves the slip mode of dislocations and fuses the dislocation more efficiently.

The laser confocal microscope (LCM) images of S21 and S22 are also shown in Fig. 5. Clear crosshatched patterns are observed in all images, indicating a good relaxation 

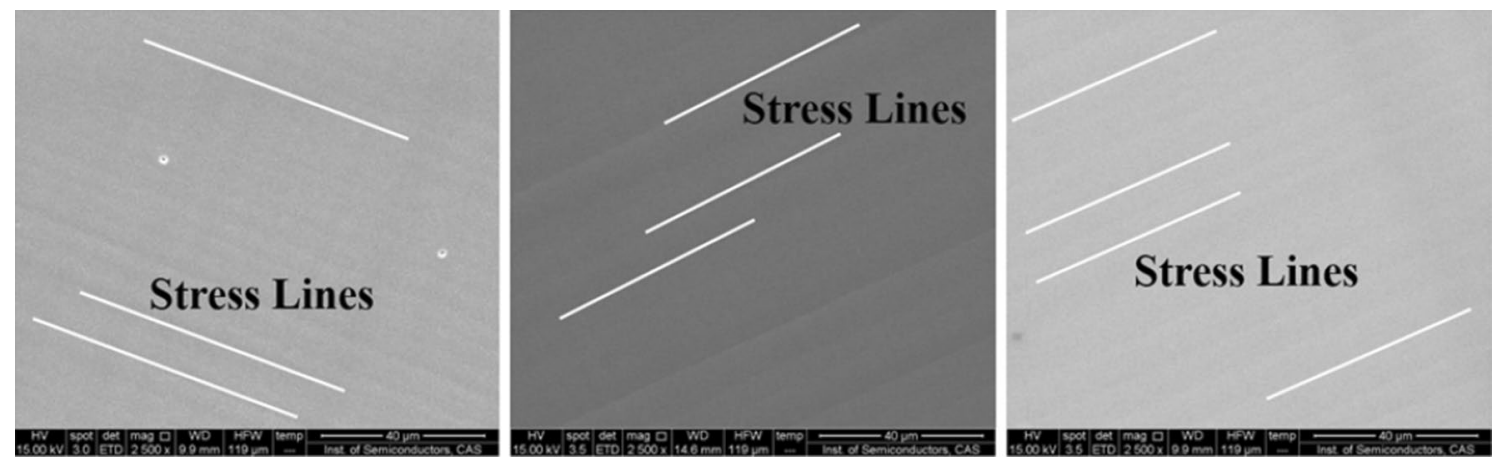

Fig. 3 SEM image of a S11 b S12 and c S13 with the magnification of 2500x

Fig. 4 AFM images of samples S21 and S22, (left) near crosshatch channel region, (right) relative flat region
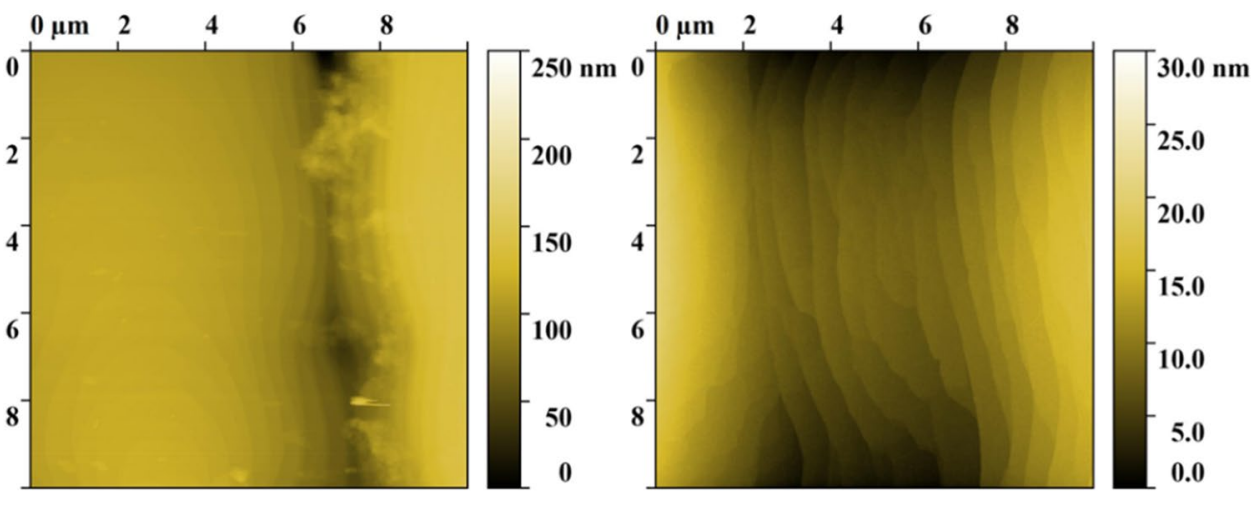

(a) $\mathrm{S} 21$

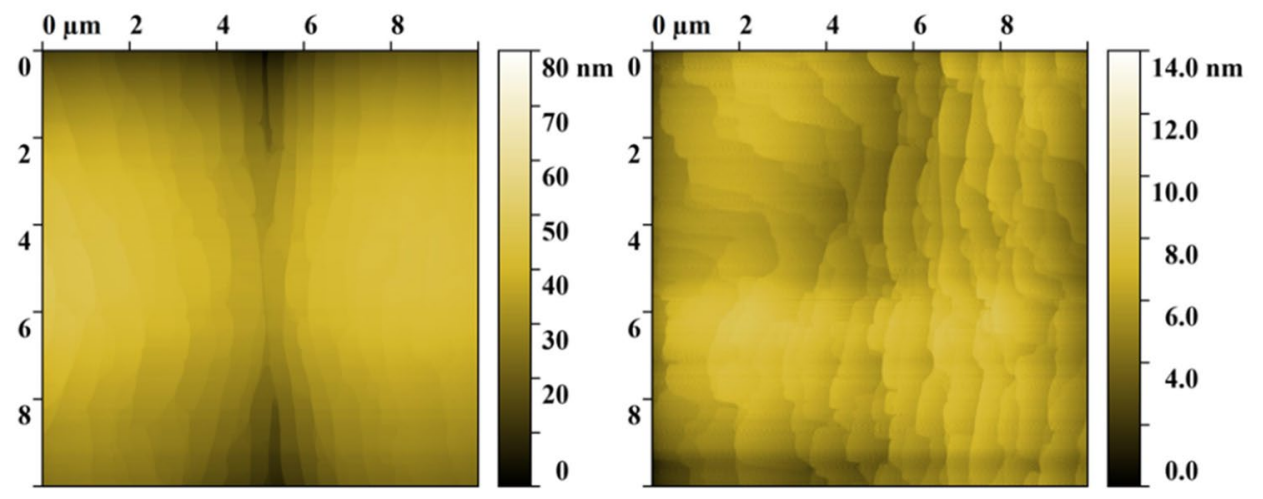

(b) $\mathrm{S} 22$ state of the large lattice mismatch in the epitaxy layers [28]. The results of the crack formation at the crosshatch pattern valleys (the colored lines in Fig. 5) show that the stress is concentrated at the valleys of the crosshatch pattern, as reported previously [29]. Moreover, Fig. 5 shows that the surface roughness of S22 $(\mathrm{Ra}=0.1949 \mu \mathrm{m})$, which is the sample with $\mathrm{Gl}$, is slightly smaller than that of the S21 $(\mathrm{Ra}=0.2031)$, which does not have $\mathrm{Gl}$.

We also obtained the asymmetric reciprocal space mappings (RSM) of S11 and S13 using high-resolution X-ray diffractometer (HRXRD). Because the epitaxy layer is thick, the asymmetric diffraction in the (115) direction was chosen to obtain further information about the lower buffer layer and substrate.

Figure $6 a$ is the RSM without adding a third axis of the HRXRD. In this case, a stronger diffraction signal can be obtained; however, the peak width of the epitaxy layer will be broadened due to the broadening of the X-ray. Because a small amount of overshoot is introduced to improve the crystal quality during the sample growth, an additional diffraction signal is observed in the region outside the epitaxy layer. The relaxation rate calculated 
Fig. 5 Laser confocal microscope image

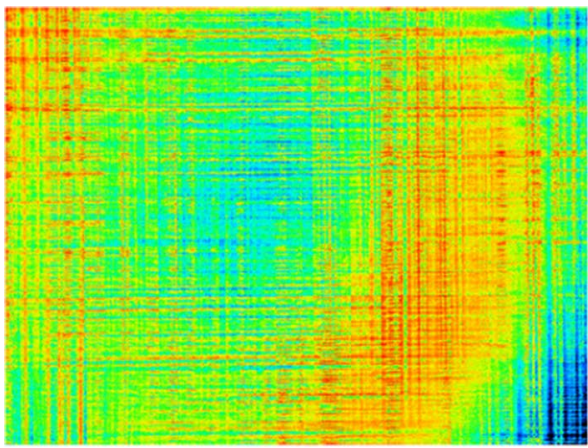

(a) $\mathrm{S} 21$

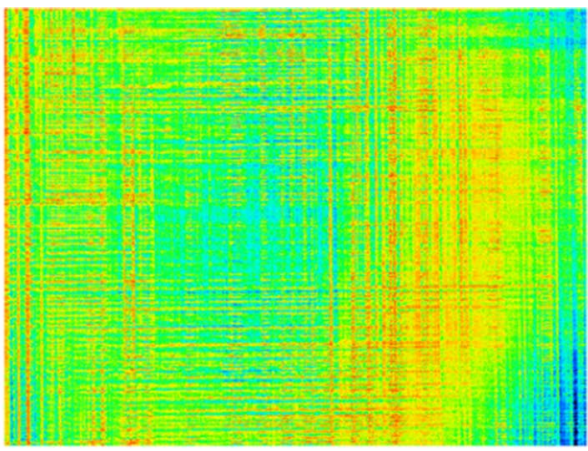

(b) S22

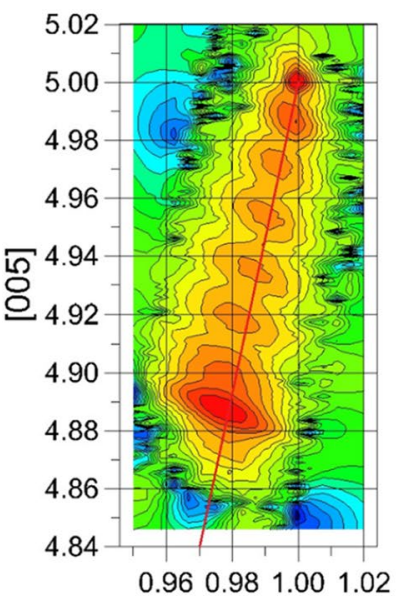

[110]

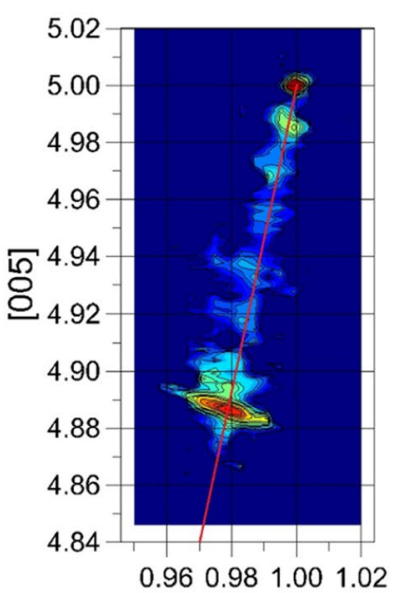

[110]
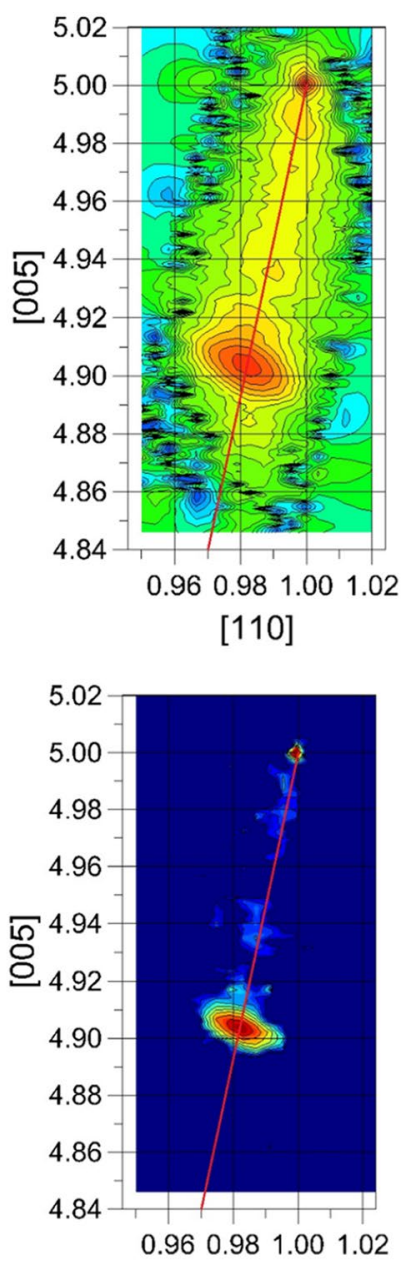

[110]
Fig. 6 Asymmetric (115) reciprocal space maps of S11 and S13 a without third axis and $\mathbf{b}$ with third axis

using software analysis in Fig. $6 \mathrm{~b}$ is $98.676 \%$ for sample $\mathrm{S} 11$ and $98.60 \%$ for sample S13, which indicates that the epitaxial layers of both samples have almost completely relaxed. Furthermore, Fig. 6 a shows that $S 11$ contains distinct buffer subpeaks while the buffer region of $\mathrm{S} 13$ does not have distinct subpeaks, which is similar to the RSM of linear-graded buffer. This is because the interface between the buffer layers becomes blurred after introducing the $\mathrm{Gl}$. A convex grade buffer glide mechanism and the pair annihilation mechanism (suggested by Choi et al. [30]) can be expressed using the following expression:

$\frac{\mathrm{d} D(x)}{\mathrm{d} x}=-a D^{2}$

where $a$ is a constant determined by the pair annihilation probability and $D$ is near-surface TD density, which, in our experiment, is assumed to be the TD density at the end of the linear change. We solve this function to obtain:

$D(x)=\frac{1}{a x+1 / D_{0}}$

where $D_{0}$ is the TD density of the previous layer. By applying $\mathrm{Gl}$, the annihilation length in the step region is increased. As a result, the TD density is reduced and the crystal quality is improved.

We also applied the PL test to the samples with and without the Gl. The PL intensity almost doubled with the introduction of $\mathrm{Gl}$ to the step-graded buffers, and the FWHM decreased, indicating an increase in the crystal quality. Using the buffer layer as a pseudosubstrate, we grew a five-period InAs/InGaAs multi-quantum well and obtained the PL spectrum at about $2.45 \mu \mathrm{m}$ at room temperature (RT $300 \mathrm{~K}$ ). This proves that the applying of the $\mathrm{Gl}$ can improve the crystal quality of the large-latticemismatch epitaxy layers (Fig. 7).

In summary, XRD, SEM, AFM, LCM, RSM, and PL tests are applied to the samples with and without GI. The introduction of GI reduces the FWHM of the epilayer peaks by more than $15 \%$ according to XRD results. Based on the SEM, LCM, and AFM test results, the surface roughness also reduces significantly after the application

\section{SN Applied Sciences}


Fig. 7 a PL test results for samples S21 and S22. b PL test results for an $\ln A s / \operatorname{lnGaAs}$ multi-quantum well structure at $300 \mathrm{~K}$

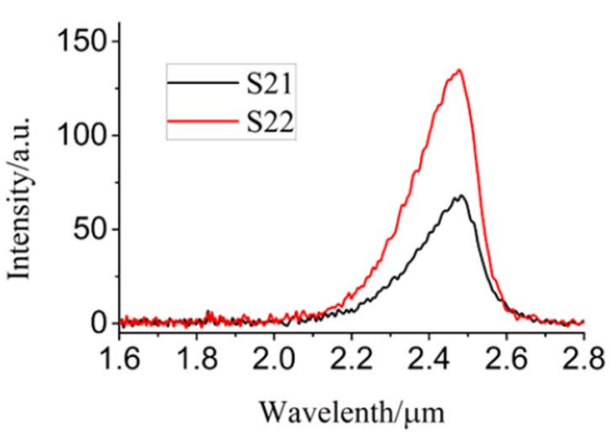

of GI. Moreover, the PL intensity of the epilayer with GI buffer is about twice as large as the one without GI buffer. The AFM and RSM results illustrate that GI buffer changes the dislocation fusion mechanism and is benefit to the epilayer growth.

\section{Conclusion}

We discussed the effect of the introduction of Gl to stepgraded buffers on the growth quality of large-lattice-mismatch materials. The $\mathrm{Gl}$ causes a continuous gliding and annihilation of the dislocation between the steps of the step-graded buffers. Using the XRD, LCM, SEM, AFM, RSM, and PL measurements and analyses, we observed that the introduction of $\mathrm{Gl}$ enhances the dislocation fusion and improves the crystal quality of the large-lattice-mismatch materials.

Acknowledgements We thank Dr. B. J. Sun and Dr. B. Qu for the useful discussion about the RSM. We thank Dr. Y. Zhang, Dr. S. H. Wei, X. L. Chai, and M. Liu for the PL, AFM, and RSM measurements. This work is supported by the National Key Research and Development Program of China 2016YFB0402400; the National Basic Research Program of China (973 Program) 2015CB932402; and the Open Fund of IPOC (BUPT) IPOC2016B006.

\section{Compliance with ethical standards}

Conflict of interest We do not have any conflicts of interest to declare. We further declare that we do not have any commercial or associative interest that represents a conflict of interest in connection with the submitted work.

\section{References}

1. Kumar R, Bag A, Mukhopadhyay P, Das S, Biswas D (2015) Comparison of different grading schemes in InGaAs metamorphic buffers on GaAs substrate: tilt dependence on cross-hatch irregularities. Appl Surf Sci 357:922-930

2. Romanato F, Napolitana E, Carnera A, Drigo AV, Lazzarini L (1999) Strain relaxation in graded composition InxGa1-xAs/GaAs buffer layers. J Appl Phys 86:4748
3. Chyi Jl, Shieh JL, Pan JW, Lin RM (1996) Material properties of compositional graded InxGa1-xAs and InxAl1-xAs epilayers grown on GaAs substrates. J Appl Phys 79:8367

4. Cordier Y, Ferre D, Chauveau JM, Dipersio J (2000) Surface morphology and strain relaxation of InAIAs buffer layers grown lattice mismatched on GaAs with inverse steps. Appl Surf Sci 166:442-445

5. D'Hondt M, Moerman I, Demeester P (1997) Characterization of $2 \%$ mismatched InGaAs and InAsP layers grown on different buffer layers and at different growth temperatures. J Cryst Growth 170:616-620

6. Hudait MK, Lin Y, Ringel SA (2009) Strain relaxation properties of InAsyP1-y metamorphic materials grown on InP substrates. J Appl Phys 105:061643

7. Kumar R, Mukhopadhyay P, Bag A, Jana SK, Chakraborty A, Das S, Mahata MK, Biswas D (2015) Comparison of different pathways in metamorphic graded buffers on GaAs substrate: indium incorporation with surface roughness. Appl Surf Sci 324:304-309

8. Haupt M, Kohler K, Ganser P, Muller S, Rothemund W (1997) Molecular beam epitaxy of Al0.48In $0.52 \mathrm{As} / \mathrm{Ga} 0.47 \mathrm{In} 0.53 \mathrm{As}$ heterostructures on metamorphic AlxGayln 1-x-yAs buffer layers. J Cryst Growth 175/176:1028-1032

9. Saha S, Cassidy DT, Tompson DA (2014) Comparison of different grading approaches in metamorphic buffers grown on a GaAs substrate. J Cryst Growth 386:183-189

10. Behet M, van der Zanden K, Borghs G (1998) Metamorphic InGaAs/InAlAs quantum well structures grown on GaAs substrates for high electron mobility transistor applications. Appl Phys Lett 73:2760

11. Hudait $M K$, Lin $Y$, Andre CL, Sinha PM, Tivarus CA, Ringel SA (2002) Relaxed InAsP layer grown on step graded InAsP buffers by solid source MBE. In: Material research society proceedings 722, K10.2. https://doi.org/10.1557/PROC-722-K10.2

12. Ji L, Lu SL, Jiang DS, Zhao YM, Tan M, Zhu YQ, Dong Jr (2013) $0.6 \mathrm{eV}$ bandgap InGaAs thermophotovoltaic devices with compositionally undulation step graded InAsP buffers. Chin Phys B 22:026802

13. Czaban JA, Thompson DA, Robinson BJ (2007) Improved InAsP metamorphic layers grown on an InP substrate using underlying InP grown at low temperatures. Semicond Sci Technol 22(4):408. https://doi.org/10.1088/0268-1242/22/4/020

14. Sacedon A, Gonzalez-Sanz F, Calleja E, Munoz E, Molina SI, Pacheco FJ, Araujo D, Garcia R, Lourenco M, Yang Z, Kidd P, Dunstan D (1995) Design of InGaAs linear graded buffer structures. Appl Phys Lett 66:3334

15. Li JP, Miao GQ, Zhang ZW, Zeng YG (2015) Experiments and analysis of the two-step growth of InGaAs on GaAs substrate. Cryst Eng Commun 17:5808

16. Liu X, Song H, Miao GQ, Jiang H, Cao LZ, Li DB, Sun XJ, Chen YR, Li ZM (2011) Effect of buffer layer annealing temperature on the 
crystalline quality of InGaAs layers grown by two step growth method. J Alloy Compd 509:6751

17. Galiev GB, Pushkarev SS, Vasilevskii IS, Zhigalina OM, Klimov EA, Zhigalina VG, Imamov RM (2013) Study of the influence of strained superlattices introduced into a metamorphic buffer on the electrophysical properties and the atomic structure of InAIAs/InGaAs MHEMT heterostructures. Semiconductors 47(4):532-537

18. Chen XY, Gu Y, Zhang YG (2017) Epitaxy and device properties of InGaAs photodetectors with relatively high lattice mismatch. Interch Epitaxy Chapter 9:203. https://doi.org/10.5772/intec hopen.70259

19. Romanov AE, Pompe W, Beltz GE, Speck JS (1996) An approach to threading dislocation reaction kinetics. Appl Phys Lett 69:3342

20. Fitzgerald EA, Kim AY, Currie MT, Langdo TA, Taraschi G, Bulsara MT (1999) Dislocation dynamics in relaxed graded composition semiconductors. Mater Sci Eng B 67:53

21. Romanov AE, Pompe W, Mathis S, Beltz GE, Speck JS (1999) Threading dislocation reduction in strained layers. J Appl Phys $85: 182$

22. Zou J (1993) Growth direction dependence on strain relief by misfit dislocations in strained layer heterostructures. Thin Solid Films 235:6

23. Seki H, Koukitu A (1986) Thermodynamic analysis of metalorganic vapor phase epitaxy of III-V alloy semiconductors. J Cryst Growth 74:172
24. Fang $X$, Gu Y, Zhang YG, Zhou L, Wang K, Li HSBY, Liu KH, Cao YY (2013) Effects of compositional overshoot on InP-based InAIAs metamorphic graded buffer. J Infrared Millim Waves 32(6):481

25. Dunn CG, Koch EF (1957) Comparison of dislocation densities of primary and secondary recrystallization grains of Si-Fe. Acta Metall 5:548

26. Yonenaga I (1998) Dynamic behavior of dislocations in InAs: in comparison with III-V compounds and other semiconductors. J Appl Phys 84:4209

27. Yonenaga I, Sumino K (1991) Dislocation velocity in indium phosphide. Appl Phys Lett 58:48

28. Albrecht M, Christiansen S, Michler J, Dorsch W, Strunk HP, Hansson PO, Bauser E (1995) Surface ripples, crosshatch pattern, and dislocation formation: cooperating mechanisms in lattice mismatch relaxation. Appl Phys Lett 67:9

29. Yoon M, Lee B, Baek JH, Park HH, Lee EH (1996) Evolution of the surface cross-hatch pattern in $\ln _{\mathrm{x}} \mathrm{Ga}_{1-\mathrm{x}} \mathrm{As} / \mathrm{GaAs}$ layers grown by metal-organic chemical vapor deposition. Appl Phys Lett 68:1

30. Choi H, Jeong Y, Cho J, Jeon MH (2009) Effectiveness of nonlinear graded buffers for $\operatorname{In}(\mathrm{Ga}, \mathrm{Al}) \mathrm{As}$ metamorphic layers grown on GaAs (001). J Cyrst Growth 311:1091

Publisher's Note Springer Nature remains neutral with regard to jurisdictional claims in published maps and institutional affiliations. 